\title{
Vibration signals denoising method based on wavelet analysis Yaru YUE ${ }^{1, a}$, Jialin ZHU ${ }^{2, b}$, Shilong $\mathrm{ZHU}^{2, \mathrm{C}}$ \\ ${ }^{1}$ College of Automation,Beijing Information Science and Technology University, Beijing,100192, China \\ ${ }^{2}$ College of Automation,Beijing Information Science and Technology University, Beijing,100192, China \\ ${ }^{3}$ College of Automation,Beijing Information Science and Technology University, Beijing,100192, China \\ aemail: yueyaru188@163.com, bemail:jlzhu@bistu.edu.cn, Cemail:zhushilong8023@163.com
}

Keywords: wavelet analysis; multi resolution; threshold; denoising; Matlab

\begin{abstract}
At present, the application of gas insulated metal enclosed switchgear (GIS) in power system is used more and more widely, but the detection of GIS is often disturbed by various kinds of noise, which affects the effective location of the fault point. Commonly it can not reflect the frequency characteristics of the signal locally for using Fourier denoising method, moreover, it is difficult to meet the requirements of the actual detection. Based on this problem, the system used the soft and hard threshold method to set the threshold value of the vibration signal. And the method of wavelet analysis with multi resolution characteristics was used to reduce the noise effectively. Through the Matlab simulation and the actual test results to verify its accuracy, it proves that denoising method based on wavelet analysis is a suitable way to extract useful signals, improve Signal to Noise Ratio and locating accuracy.
\end{abstract}

\section{Introduction}

Gas insulated metal enclosed switchgear(GIS) is widely used in the field of high voltage transmission because of its small size, good insulation properties and high reliability. But GIS has strong electromagnetic waves and white noise around the equipment, such as thermal noise of equipment, noise in ground grid, dynamic power line of equipment, relay protection circuit and so on, which seriously affect the extraction of flashover characteristic signal[1]. In general, noise is often produced during the generation or transmission of a signal, so we capture the signal that contains noise from the sensors[2]. The addition of noise will seriously affect the quality of the signal, and also increase the difficulty of processing the follow-up signal, which will greatly reduce the timeliness of positioning and accuracy. In general, to eliminate noise interference can use the hardware suppression methods, such as using a variety of filter circuits and shielding measures; and can also use the software processing methods, which use the signal noise separation algorithm to eliminate noise[3-4].

In practical application, signal always contains part of spike or mutation, and the noise is not necessarily stationary white noise. Using the traditional Fourier method to analyze this kind of effect is not obvious, because it does not show a signal change at a point in time[5]. Besides, weak signal that has the obvious changes in a certain time and space will affect the whole spectrum of the signal[6]. The system using wavelet method for reducing the noise of the signal, wavelet analysis is a time and frequency analysis method, can be used to do multi resolution analyze for the signal in time and frequency domain, and can distinguish the abrupt part and the noise in the signal effectively[7-9]. It can be used to extract the transient signal and the waveform characteristic of the extracted signal effectively from the non-stationary vibration signal with different resolutions in the time-frequency plane[10-11]. Here, GIS flashover fault detection system based on wavelet analysis use threshold denoising method that can reduce the white noise and electromagnetic wave in the environment, and greatly improve the Signal to Noise Ratio and accuracy of positioning[12]. 


\section{Wavelet Analysis Theory}

A square integrable function $f(t) \in L^{2}(R)$ can be regarded as a function of step by step approximation. Each level of approximation with a low-pass smoothing function $\psi(t)$ for $f(t)$ is a stage telescopic result. Doing two level decomposition for a space will produce a group step by step contained subspaces:

$$
\ldots, \quad V_{0}=V_{1} \oplus W_{1}, \quad V_{1}=V_{2} \oplus W_{2}, \ldots, \quad V_{j}=V_{j+1} \oplus W_{j+1}, \ldots \quad j \in[-\infty,+\infty]
$$

A smaller $j$ value is a greater space.

The $v_{j}$ space satisfies representation of the following multiresolution:

$$
f_{j}=f_{j-1}+d_{j-1}=f_{j-2}+d_{j-2}+d_{j-1}=f_{M}+d_{M}+d_{M+1}+\cdots d_{j-1}
$$

Where,

$$
\begin{aligned}
& f_{l}(t)=\sum_{k \in Z} c_{k}^{l} \varphi_{l, k}(t) \in V_{l}, \\
& d_{l}(t)=\sum_{k \in Z} d_{k}^{l} \psi_{l, k}(t) \in W_{l}, \quad l=M, \cdots, j-1
\end{aligned}
$$

$f_{l}(t)$ expresses low frequency components of $f_{j}, d_{l}(t)$ expresses the high frequency components of $f_{j}$.

The orthogonal projection $f_{j} \square$ is known in $V_{j}$, then $f_{l}(t)$ and $d_{l}(t)$ in scale function $\left\{c_{k}^{j}\right\}$ and wavelet coefficients $\left\{d_{k}^{j}\right\}$ can be represented by the following formula:

$$
f_{j}(t)=\sum_{k \in Z} c_{k}^{j} \varphi_{j, k}(t)=\sum_{k \in Z} c_{k}^{j-1} \varphi_{j-1, k}(t)+\sum_{k \in Z} d_{k}^{j-1} \psi_{j-1, k}(t)
$$

Hypothesis observated signal is expressed as:

$$
y_{i}=x_{i}+e z_{i}
$$

In the formula, $x_{i}$ is a useful signal, $e$ is noise level, $z_{i}$ is noise signal. The $z_{i}$ obeys the normal distribution $N:\left(0, \sigma_{\pi}^{2}\right)$.

Besides, image using the soft threshold method is smoother, but image on the edge may appear distortion; and the hard threshold method has a better marginal local characteristic. Therefore, it is necessary to design a kind of the eclectic method of soft threshold and hard threshold. This method can get approximate optimal estimation of the useful signal, the function expression:

$$
f_{h}(t)=\left\{\begin{array}{cc}
\operatorname{sgn}(x(t))(|x(t)|-\lambda a) & |x(t)|>a \\
0 & |x(t)| \leq a
\end{array}\right.
$$

In the formula, $\square \lambda$ is an arbitrary constant, $0<\lambda \leq 1$, through specific denoising effect to determine this value.

\section{Simulation analysis}

Firstly, the signal features of white noise were extracted from the GIS simulation equipment. Oscillograph was shown in Figure 1.

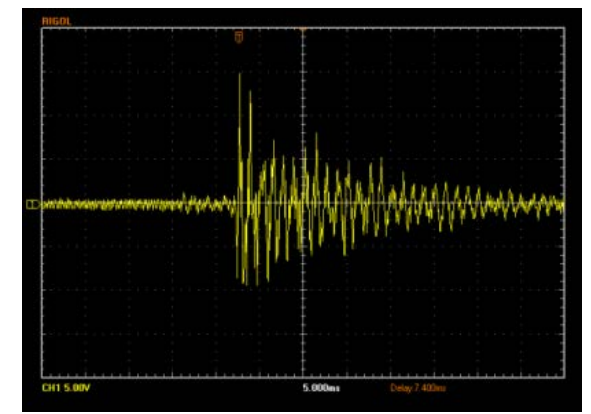

Fig. 1 analog signal acquisition

Then, as could be seen from the extracted signals, the acoustic signal was similar to the oscillator signal. Therefore, a set of mathematical model was established in the experimental process, namely exponential decay model. 
Single exponential decay model:

$f(t)=a_{1}^{-t / T_{1}} \sin \left(f_{c} \times 2 \pi t\right)$

Double exponential decay oscillator model:

$$
f(t)=a_{2}\left(e^{-2.2 t / T_{2}}-e^{-1.3 t / T_{3}}\right) \sin \left(f_{c} \times 2 \pi t\right)
$$

In the formula, $a_{1}$ and $a_{2}$ are the amplitude of discharge signal, $T_{1} 、 T_{2}$ and $T_{3}$ are the decay time of the signal, $f_{c}$ is oscillation frequency of the signal .

Simulation experiments used the combination of two models in the formula. By MATLAB7.10 simulation experiment, the parameters were set for the $f_{c}=5 \times 10^{5} \mathrm{~Hz}, \quad f_{s}=1 \times 10^{7} \mathrm{~Hz}$, as shown in Figure 2.

In the simulation signal by adding the noise of a certain Signal to Noise Ratio, as shown in Figure 3.

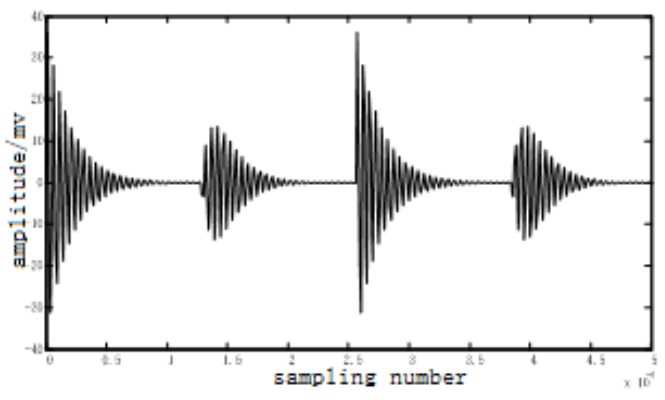

Fig. 2 simulation signal

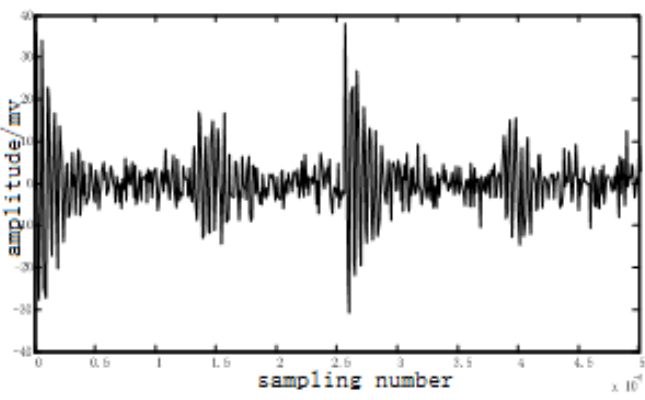

Fig. 3 simulation signal with noise

In the experiment, the correlation were introduced to judge the quality of signal processed.

The coif wavelet system using soft and hard threshold method, selecting different wavelet functions and decomposition levels, got the correlation coefficient table, as shown in table 1. From the table 1 can be seen, when decomposition layer is 6 , wavelet function is coif5, correlation coefficient is the biggest, and the corresponding value is 0.8931 . Denoising effect is the best.

Table 1 the correlation coefficient of coif wavelet using soft and hard threshold denoising method

\begin{tabular}{cccccc}
\hline & coif1 & coif2 & coif3 & coif4 & coif5 \\
\hline 1 & 0.8130 & 0.8480 & 0.8705 & 0.8823 & 0.8924 \\
\hline 2 & 0.7712 & 0.8038 & 0.8311 & 0.8413 & 0.8507 \\
\hline 3 & 0.7916 & 0.8281 & 0.8536 & 0.8642 & 0.8713 \\
\hline 4 & 0.8010 & 0.8379 & 0.8638 & 0.8650 & 0.8815 \\
\hline 5 & 0.8076 & 0.8475 & 0.8745 & 0.8836 & 0.8895 \\
\hline 6 & 0.8089 & 0.8497 & 0.8771 & 0.8869 & 0.8931 \\
\hline 7 & 0.8089 & 0.8503 & 0.8774 & 0.8865 & 0.8925
\end{tabular}

This scheme uses coif5 wavelet that decomposition layer is 6 . simulation result is shown in Figure 4.

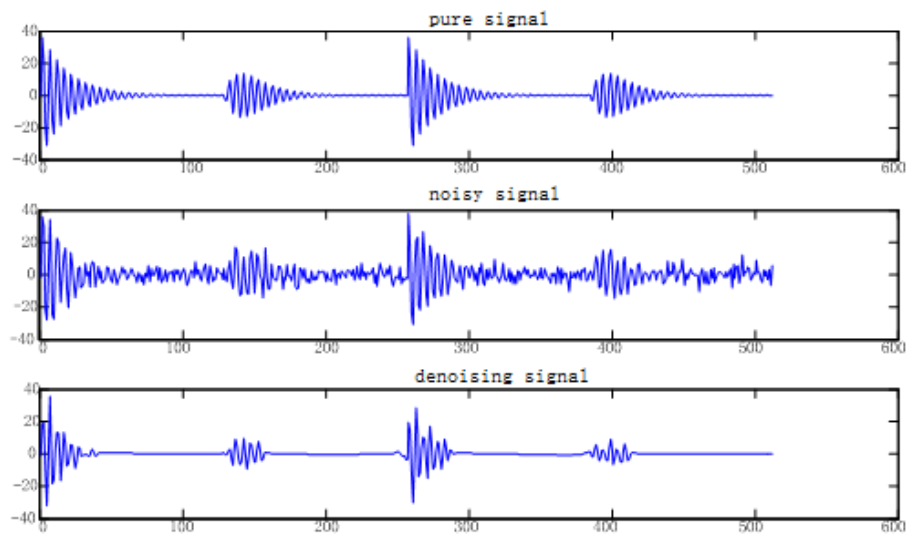

Fig. 4 simulation signal processing 


\section{Test results}

The sensors are stuck in the middle of the outer wall of the air chamber, and then the same position of the wall is struck, and the position of the sound box is continuously moved, as shown in Figure 5. In the course of the experiment, the knocked position is shown in Figure 6.

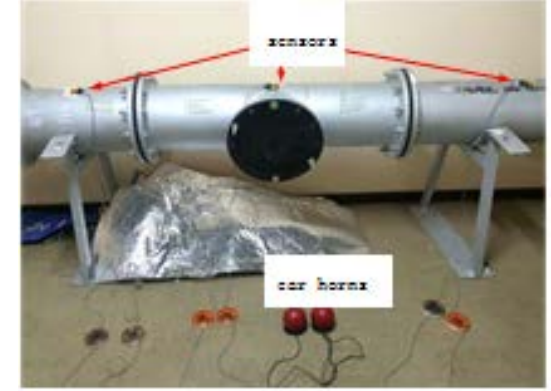

Fig. 5 experimental platform

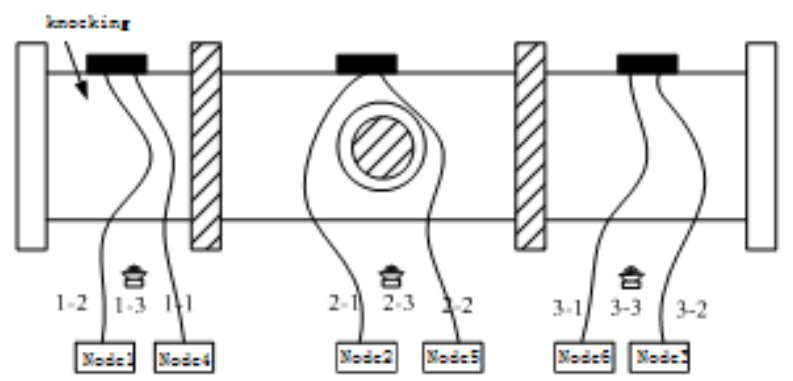

Fig. 6 schematic diagram of the test platform.

First determine the location of a hit, as shown in Figure 6. In a quiet environment, using the equipment without adding wavelet algorithm ( Node1,Node2,Node3) carries out three tests, the test data is shown in Table 2.

\begin{tabular}{cccc}
\multicolumn{4}{c}{ Table 2 test data before adding noise } \\
\hline $\begin{array}{c}\text { Sequence } \\
\text { number }\end{array}$ & Node 1 & Node 2 & Node 3 \\
\hline 1 & 958 & 730 & 318 \\
2 & 756 & 346 & - \\
3 & 902 & 687 & 358 \\
\hline
\end{tabular}

Then, according to the sequence of the sound box in Figure 6, it is placed(The speaker is placed in one of the locations at each test.), and then hit three times in each position, finally two sets of test data are recorded, which are shown in Table 3 and table 4.

Table3 Using wavelet analysis before the test results Table4 Test results by using the method of wavelet analysis

\begin{tabular}{cccc}
\hline $\begin{array}{c}\text { Speaker } \\
\text { position }\end{array}$ & Node 1 & Node 2 & Node 3 \\
\hline $1 \# 1$ & 1636 & 1224 & 544 \\
$1 \# 2$ & 1422 & 1357 & 672 \\
$1 \# 3$ & 1484 & 1383 & 563 \\
$2 \# 1$ & 1936 & 2043 & 621 \\
$2 \# 2$ & 1926 & 1861 & 431 \\
$2 \# 3$ & 1513 & 1322 & 800 \\
$3 \# 1$ & 1523 & 1250 & 1311 \\
$3 \# 2$ & 1663 & 1378 & 1193 \\
$3 \# 3$ & 1499 & 1032 & 1096 \\
\hline
\end{tabular}

\begin{tabular}{cccc}
\hline $\begin{array}{c}\text { Speaker } \\
\text { position }\end{array}$ & Node 4 & Node 5 & Node 6 \\
\hline $1 \# 1$ & 1012 & 700 & 232 \\
$1 \# 2$ & 857 & 662 & - \\
$1 \# 3$ & 826 & 673 & 201 \\
$2 \# 1$ & 936 & 755 & 332 \\
$2 \# 2$ & 910 & 635 & 267 \\
$2 \# 3$ & 892 & 621 & 349 \\
$3 \# 1$ & 893 & 696 & 258 \\
$3 \# 2$ & 758 & 401 & - \\
$3 \# 3$ & 830 & 423 & 211 \\
\hline
\end{tabular}

From table 3 that when the sound box is placed on the 1\# position, the data of No. 1 node and No. 2 node vary little; when the sound box is placed on the 2\# position, sometimes the data of No. 2 node are slightly greater than the data of No. 1 node, and the data of No. 1 and No. 2 node are basically similar; when the sound box is placed on the 3\# position, the signal data of No. 2 and No. 3 node basically have not difference, which obviously no longer meet the law of attenuation. And the data in table 4 are basically in accordance with the data characteristics of table 2, which illustrates that basically meets attenuation characteristics of the signal in the GIS equipment. Some of the data also exist certain deviations, maybe due to the existence of differences among equipment lead to deviations of the data. This shows that the noise in the environment will affect the accuracy of positioning in some cases. And after joining the wavelet analysis algorithm, through the comparison between the experimental data, we can still find out the position of flashover, and can basically be able to respond to the strength of the signal. 


\section{Conclusion}

The results of simulation and experiment show that denoising method based on wavelet analysis is a superior method to extract the useful signal, remove the noise and reflect the mutation signal, which has very wide application prospect and practical value. In the detection of gas insulated metal enclosed switchgear, the traditional signal denoising method has been unable to feed usually requires, where, in this paper, the wavelet denoising method is suitable for detecting stationary target signal with the transient abnormal phenomena. What's more, it retains the variational components, and can completely extract the feature of the part signal, and greatly improves the signal-to-noise ratio of the system and accuracy and timeliness of the detection.

\section{References}

[1] Zhu Shilong, Zhu Jialin, Chen Fubin. Development of the Differential Amplifier which is used to reject common-mode signal. DEStech Publication[J]. 2015,9:984-990.

[2] Jong-Soo Choi. Aerodynamic noise generation in centrifugal turbomachinery. KSME Journal[J]. 1994, Vol.8 (2):161-174.

[3] L. Kaur, S. Gupta, R.C. Chauhan. Image denoising using wavelet thresholding. Comput. Vis. Graph. Image Process [J]. 2002.

[4] S.G. Chang, B. Yu, M. Vetterli. Adaptive wavelet thresholding for image denoising and compression. IEEE Trans. Image Process [J]. 2000,9 (9) :1535-1545.

[5] Bing Yue, and so on. An anomaly intrusion detection method using fourier transform. Journal of Electronics (China)[J]. 2004, Vol.21 (2).

[6] Wolfgang Stefan, and so on. Sparsity Enforcing Edge Detection Method for Blurred and Noisy Fourier data. J. Sci. Comput[J]. 2012, Vol.50.

[7] Yu Guangbin, and so on. Reducer vibration de-noising signal research based on wavelet analysis theory. Journal of Convergence Information Technology[J]. 2013, Vol.8 (10):879-886.

[8] Hao Yong, Chen Bin, Zhu Rui. Analysis of several methods for wavelet denoising used in near infrared spectrum pretreatment. Guang Pu Xue Yu Guang Pu Fen Xi/Spectroscopy and Spectral Analysis[J]. 2007, 26(10).

[9] Ren S, Gao L. Simultaneous quantitative analysis of overlapping spectrophotometric signals using wavelet multiresolution analysis and partial least squares. Talanta[J]. 2008, 50(6).

[10] P.V. Kasambe, S.S.Rathod. VLSI Wavelet Based Denoising of PPG Signal. Procedia Computer Science [J]. 2015,49:282-288.

[11] Seongjun Lee, Jonghoon Kim. Discrete wavelet transform-based denoising technique for advanced state-of-charge estimator of a lithium-ion battery in electric vehicles. Energy[J]. 2015,83:462-473.

[12] Shuxin Wang, and so on. Denoising method for shear probe signal based on wavelet thresholding. Transactions of Tianjin University[J]. 2012, Vol.18 (2):135-140. 